\title{
Integrated enzymatic catalysis for biomass deconstruction: a partnership for a sustainable future
}

\author{
Sudipta De $1^{1^{*}}$ and Rafael Luque $2^{2^{*}}$
}

\begin{abstract}
Deconstruction of lignocellulosic biomass using enzymatic catalysis can offer several advantages as compared to chemical catalysis in terms of product selectivity, production cost and sustainability issues. This contribution aims to provide an account of current developments in the understanding of plant biomass microstructures and the impact of various enzymatic processes on cellulose decrystallization. Critical problems, including biomass recalcitrance, and operational factors, including potential solutions to improve their effectiveness as alternatives in future biorefineries, will be also discussed.
\end{abstract}

Keywords: Lignocellulosics, Enzyme catalysis, Cascade reactions, Heterogeneous immobilization

Plant cell walls represent a largely abundant source of renewable carbon in the biosphere. The inherent complexity of fractions including cellulose and lignin requires several deconstruction strategies to release structural polysaccharides for suitable applications. Cellulose and chitin are two highly abundant biopolymer resources with a significant physicochemical recalcitrance, which limits their rapid and cost-effective degradation. Plants indeed evolved considerable defense mechanisms against deconstruction of their cell wall polysaccharides into sugars, and, as such, lignocellulosics depolymerization to various simple fractions (e.g. sugars) for subsequent biological or catalytic conversion to fuels has been the focus of intensive research endeavors in past years.

Recent biocatalytic attempts to valorize lignocellulosic biomass for the production of chemicals and biofuels have focused on identifying enzymes with enhanced hydrolytic capabilities [1]. In nature, many microorganisms produce "free enzyme" cocktails of individual enzymes that work synergistically to depolymerize biomass [2-4]. Cellulolytic and hemicellulolytic enzymes able to deconstruct celluloses and hemicelluloses into fermentable

\footnotetext{
*Correspondence: sudiptade22@gmail.com; q62alsor@uco.es

${ }^{1}$ Laboratory of Catalysis, Department of Chemistry, University of Delhi, North Campus, Delhi 110007, India

²Departamento de Quimica Organica, Universidad de Cordoba, Campus de Rabanales, Edificio Marie Curie (C-3), Ctra Nnal IV-A, Km 396, 14014 Cordoba, Spain
}

sugars can facilitate the use of a plentiful source of renewable carbon.

Enzymatic cellulose decomposition primarily relies on glycoside hydrolases (GHs) and oxidative enzymes. GHs catalyze the cleavage of glycosidic linkages located between adjacent carbohydrate residues. Figure 1 illustrates the complementary actions of different cellulases (A) and hemicellulases (B) on the enzymatic degradation of cellulose and hemicelluloses respectively. The microstructure and treatment of plant biomass using different hydrolase enzymes, as well as understating the role of active site structures from hydrolytic and oxidative enzymes (and their overall impact on the biomass degradation process), is of utmost importance.

Scientists from National Renewable Energy Laboratory (NREL), USA have significantly contributed to advances in the field of biological conversion of lignocellulosic feedstocks into liquid fuels in the past decades. Between 1999 and 2012, NREL conducted extensive studies to quantify the economic implications associated with measured conversion-performance for the biochemical production of cellulosic ethanol. A goal for pilot-scale demonstration by 2012 of biochemical ethanol production was set at a price competitive with petroleum gasoline, which was successfully achieved through NREL's 2012 pilot plant demonstration runs. 


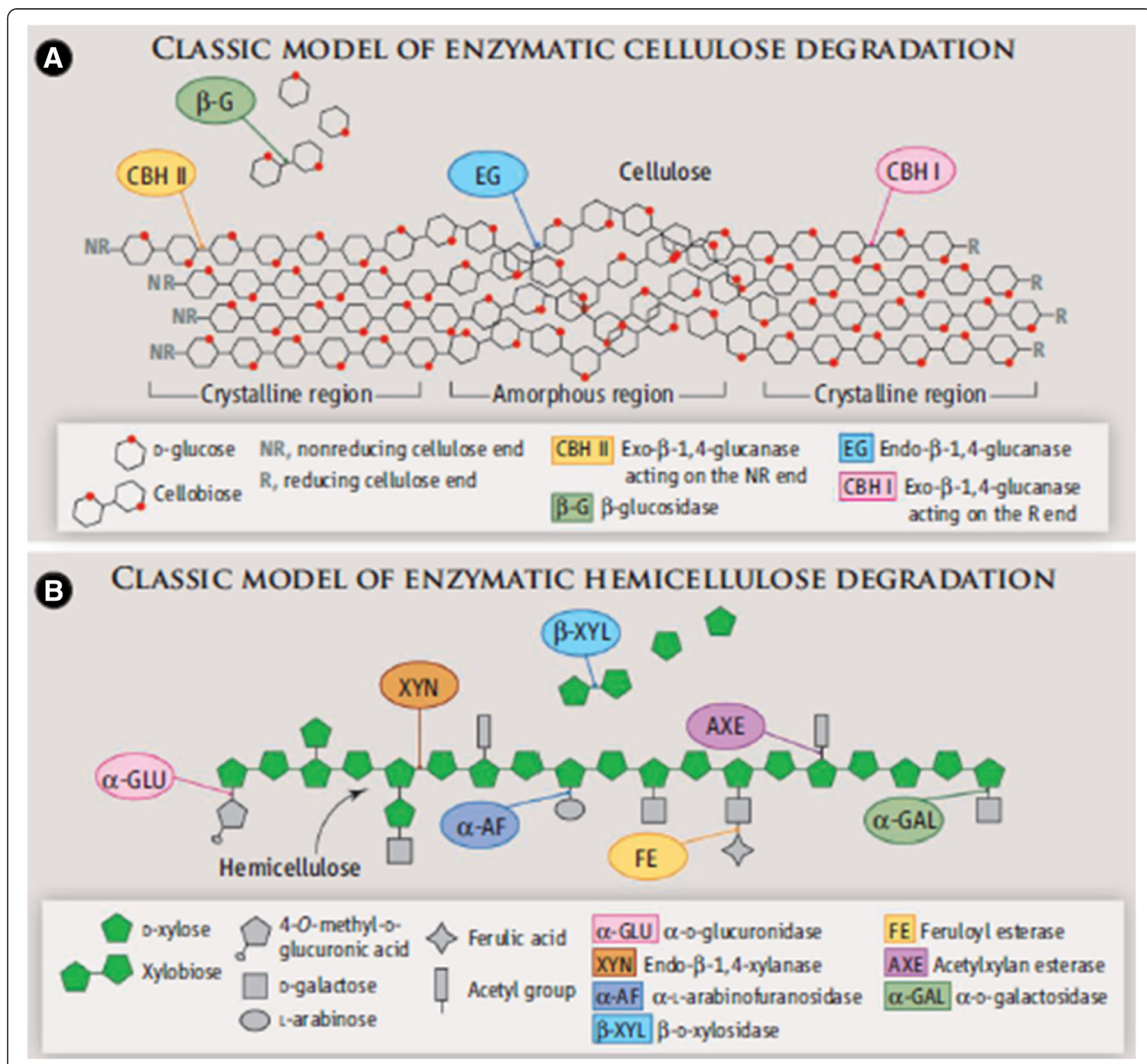

Figure 1 Classic models of enzymatic degradation of (A) cellulose and (B) hemicellulose show how plant polysaccharides are broken down. [Reproduced from Ref [3] with permission, copyright AAAS, 2013].

\section{Why enzymatic deconstruction?}

Three major approaches have been widely explored in cellulosic conversion: physical (e.g. high temperatures, pressures and various pre-treatment/conversion technologies such as milling), chemical (e.g. strong acid treatment) and biological procedures. Physical and chemical processes are generally more efficient in terms of total conversion but inherently energy consuming, with a large generation of by-products. Comparatively, enzyme-based biological processes can be performed under mild conditions with high specificity for the target product. Enzyme-assisted cellulosic conversion can therefore constitute an alternative green approach, which can potentially reduce experimental costs, reduce the formation of unwanted byproducts as well as enhance reaction efficiency (under optimum conditions) and specificity.

The use of one-pot protocols mediated by multiple enzymes without any need for isolation steps (so-called cascade reactions) and/or continuous flow processes can offer significant benefits with respect to physic-chemical conversion processes. These include decreased unit operations, decreased reactor volume, increased volumetric and space-time yields, and shortened cycle times, as well as inherent advantages of flow reactions such as simpler work-up and more controllable reaction conditions [5]. Multi-enzyme systems are complex in nature, but 
unique synergistic effects and the coupling of steps may even push unfavorable equilibria towards the formation of desired products under cascade-type processes (i.e. continuous flow conditions). However, major problems of cascading enzymatic reactions need to be overcome, including a generally lower stability and recyclability of the enzymes (as different enzymes are used under different reaction conditions). Heterogeneous immobilization techniques can overcome these issues, which will be discussed later.

\section{Pretreatment of lignocellulosics}

Enzymes must work directly at the solid-liquid interface for the depolymerization of individual cellulose chains to hydrolyze carbohydrate polymers, due to the complex composition of rigid cell walls. The surface ablation process proceeds in a slower reaction rate than any freely diffusing enzymatic reactions due to limited substrate accessibility [6]. Importantly, enzymatic hydrolysis yields are typically low ( $20 \%$ of glucan in feedstock) in the absence of pretreatment [7]. A pretreatment step is highly essential to open up the structure of hydrolysisresistant lignocellulosic matrices to enzymatic or microbial biocatalysts able to convert the carbohydrates into soluble sugars. Another major concern relates to the removal of the lignin fraction (cross-linked with the polysaccharides to make a rigid hydrophobic network) as lignin was proved to play an important role as an inhibitor in enzymatic hydrolysis, fermentation and other downstream processes [8]. Combined steps of pretreatment and enzymatic hydrolysis can easily overcome the biomass recalcitrance during a biochemical conversion process.

Dilute sulfuric acid pretreatment has been an extensively utilized pre-treatment to break down cellulosic structures. Recently, ionic liquids (ILs) were explored as novel pretreatment to effectively disrupt cellulose crystallinity [9]. However, a major issue in an integrated process relates to the recovery of ILs and isolation of the dissolved lignin-hemicellulose after pretreatment as

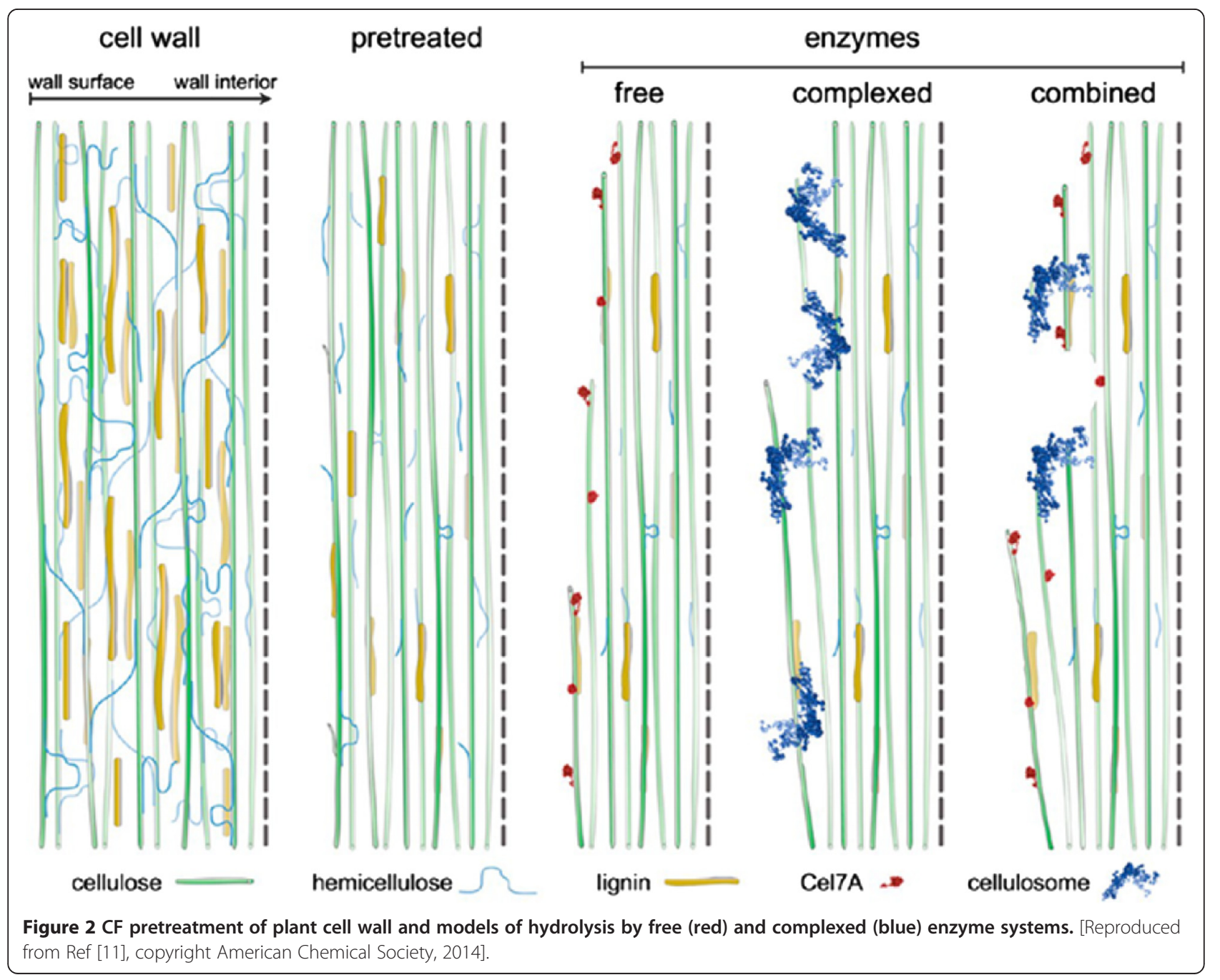


native cellulases are severely inhibited by trace amounts of residual ILs. More research is consequently needed to develop IL-tolerant enzymes as well as to achieve clear knowledge about cellulase activity, stability and structureactivity relationships in cellulose-dissolving ILs. Interestingly, some relevant advances have recently been made in discovering and developing cellulases and other glycosyl hydrolases with increased IL-tolerance [10]. Besides, more research on designing enzyme-compatible cellulosedissolving ILs and cellulose stabilization techniques have also been the focus of recent studies.

Another efficient method for lignocellulosics pretreatment is the clean fractionation (CF) technique which involves an organosolv pretreatment method utilizing water, MIBK and either acetone or ethanol with catalytic amounts of sulfuric acid to fractionate biomass components [11]. CF mainly concentrates the insoluble cellulose-enriched fraction from biomass by removing hemicellulose and lignin fractions [Figure 2] and reduces the amount of enzyme required in depolymeriztiaon of polysaccharides relative to deacetylated dilute acid pretreatment.

\section{Immobilized catalytic systems}

A critical problem of cellulases as biocatalysts relates to their quick deactivation by environmental factors (e.g. temperature), which greatly hinders their practical uses in industry. Immobilization of cellulases on suitable solid materials (i.e. amorphous or mesoporous silica, agarose gel etc.) has been reported to improve their stability and reusability without significantly reducing their catalytic activity [12-14]. Based on previous studies, mesoporous silica materials have attracted a great deal of attention due to their large specific surface area, high mechanical strength and tunable surface functionalities [15]. The cellulase activity has been shown to be largely dependent on pore size and surface area of the support, different immobilization methods (i.e. physical adsorption and chemical binding), loading amount and stability of cellulase after immobilization. Trichoderma reesei cellulases

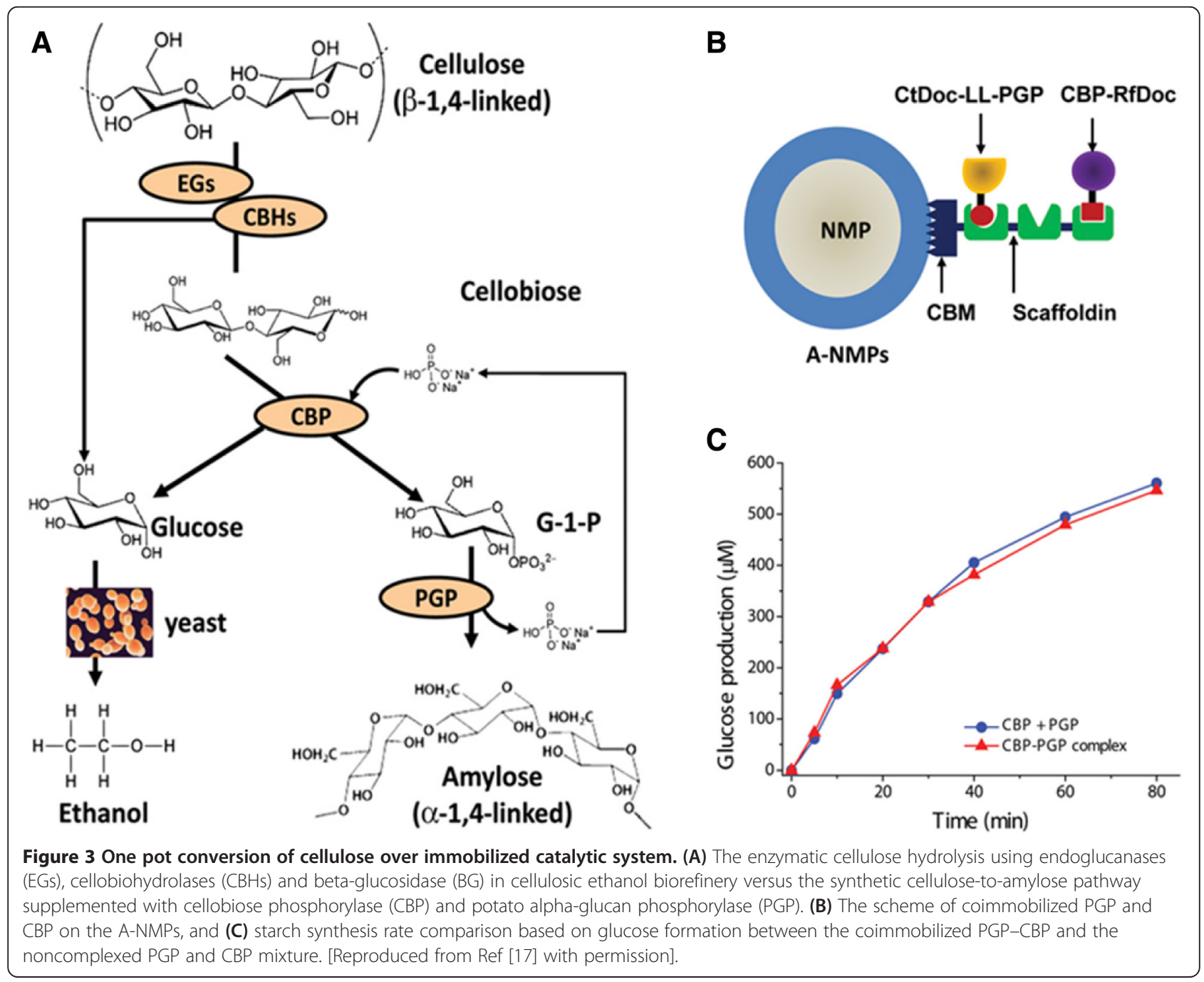


chemically bound to various supports, including silicas and magnetic nanoparticles, exhibited excellent stability and catalytic activity, exceeding $80 \%$ glucose yield from biomass [16]. Recent research efforts also disclosed an integrated sequential enzyme cascade technique to deconstruct cellulose into 5-hydroxymethylfurfural (HMF) in high yields (46.1\%).

Recently, You et al. developed a simple enzyme purification and co-immobilization process using Avicel-containing nanomagnetic particles (A-NMPs) [17]. Cellulose was transformed into amylose in a one-pot reaction through the formation of cellobiose intermediate using this immobilized catalytic system [Figure 3]. The immobilized cellobiohydrolase (CBP)-potato alpha-glucan phosphorylase (PGP) enzyme complex on A-NMPs exhibited comparable reaction rates to those of non-complexed CBP and PGP mixtures which suggested a synergetic co-immobilization could be conducted without influencing the enzymatic activity in the biocatalytic systems. The production costs in terms of cost per kilogram of enzyme is low as the combined cellulose-hydrolyzing and starch-synthesizing enzymatic mixture could easily enhance the total turnover number
(TON) values without involving any labile coenzymes (e.g. CoA and $\operatorname{NAD}(\mathrm{P})$ ). The proposed cascading enzymatic process could be highly acceptable in a potential large-scale implementation of cellulose-to-starch in future biorefineries.

The mechanism of action of many carbohydrate-active enzymes and their corresponding families derived from bacteria and fungi for the degradation of plant polysaccharides has been recently revealed $[18,19]$. Brunecky et al. have showed that CelA, an unusually large secreted multidomain cellulase from thermophilic bacteria Caldicellulosiruptor bescii, could efficiently break down recalcitrant microcrystalline cellulose at a faster rate as compared to an enzyme mixture commonly used in commercial biomass enzyme products [20]. Morphological studies of CelA-incubated cellulose suggested that CelA not only exploits the common surface ablation mechanism driven by general cellulase processivity, but also excavates extensive cavities into the surface of the substrate [Figure 4]. Interestingly, CelA showed higher specific activity on raw biomass (even on its own) as compared to physicochemically

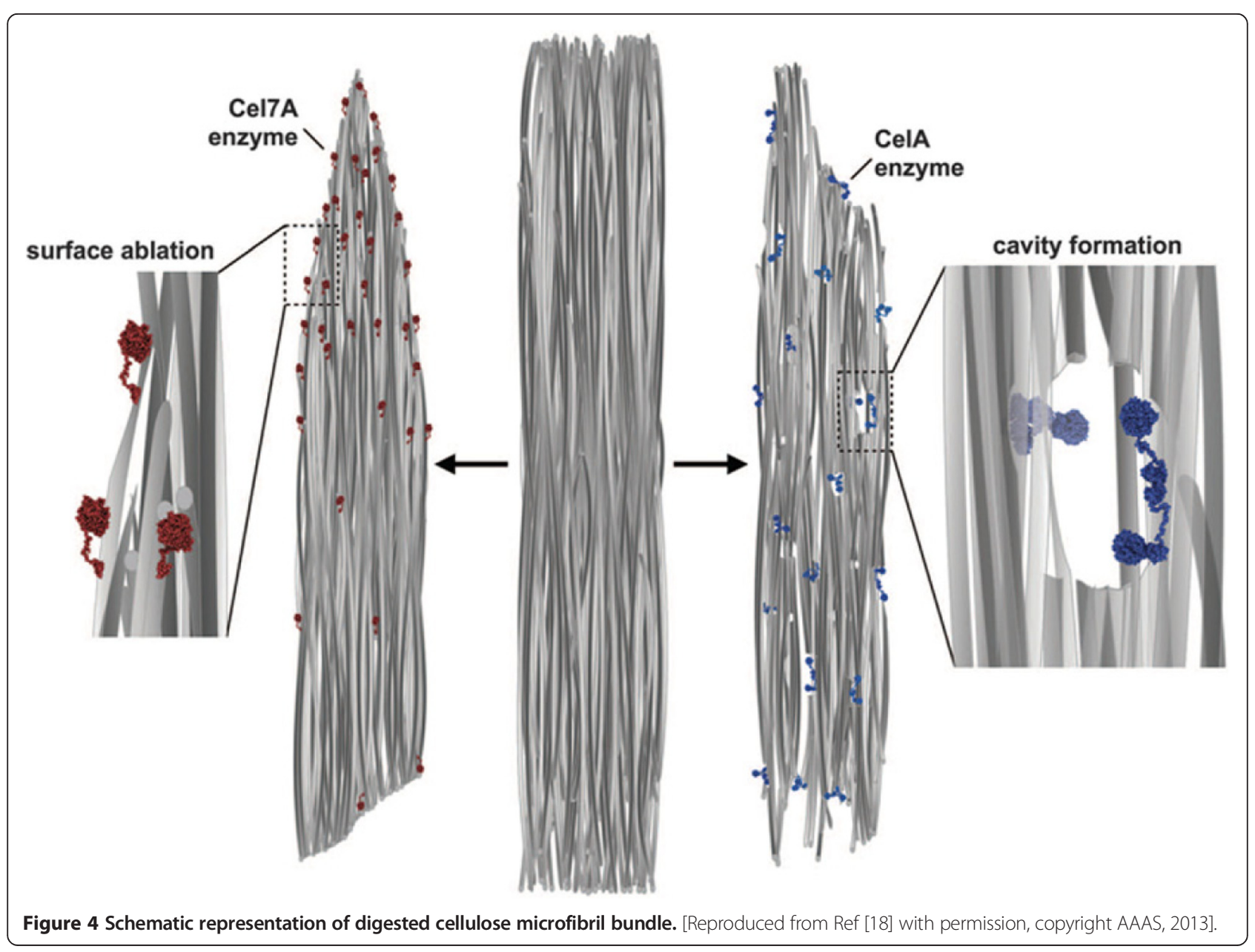


pretreated biomass. These studies could be a turning point in the development of future bioconversion technologies that will not require biomass pretreatment or the participation of a large number of enzyme components.

Despite all these advancements, the enzymatic decrystallization process is still critical and poorly understood in many cases, and thus considerable research is needed to enhance the performance of cellulase action. The mechanism of action of hydrolase enzymes in the context of the cellulose surface must be understood at the molecular level. Emerging technologies such as cascading techniques should be further investigated for more efficient biocatalytic conversions. New techniques in the microscale are also required to be developed for the quantitative large-scale screening of enzyme libraries for biomass hydrolysis. Numerous biopolymers and enzymatic processes exist in nature; we just need to find out the most effective processes and technologies with highest degree of compatibility between substrates and enzymes in terms of enzymatic action.

\section{Competing interests}

The authors declare that they have no competing interests.

\section{Authors' contributions}

Both SD and RL contributed to the writing of the manuscript, being SD main contributor of this work. Both authors read and approved the final manuscript.

\section{Acknowledgements \\ Sudipta De gratefully acknowledges the University Grants Commission (UGC), India and University of Delhi for the financial support and necessary journal access during this work. Rafael Luque gratefully acknowledges Consejeria de Ciencia e Innovacion, Junta de Andalucia for funding under project P10-FQM-6711.}

Received: 1 December 2014 Accepted: 17 March 2015

Published online: 27 March 2015

\section{References}

1. Himmel ME, Ding SY, Johnson DK, Adney WS, Nimlos MR, Brady JW, et al. Biomass recalcitrance: engineering plants and enzymes for biofuels production. Science. 2007;315:804-7.

2. Fontes CMGA, Gilbert HJ. Cellulosomes: highly efficient nanomachines designed to deconstruct plant cell wall complex carbohydrates. Annu Rev Biochem. 2010;79:655-81.

3. Berlin A. No barriers to cellulose breakdown. Science. 2013:342:1454-6.

4. Dutta S, Wu KCW. Enzymatic breakdown of biomass: enzyme active sites, immobilization, and biofuel production. Green Chem. 2014;16:4615-26.

5. Ricca E, Brucher B, Schrittwieser JH. Multi-enzymatic cascade reactions: overview and perspectives. Adv Synth Catal. 2011;353:2239-62.

6. Jeoh T, Ishizawa Cl, Davis MF, Himmel ME, Adney WS, Johnson DK. Cellulase digestibility of pretreated biomass is limited by cellulose accessibility. Biotechnol Bioeng. 2007;98:112-22.

7. Zhang YHP, Lynd LR. Toward an aggregated understanding of enzymatic hydrolysis of cellulose: noncomplexed cellulase systems. Biotechnol Bioeng. 2004;88:797-824.

8. Zeng Y, Zhao S, Yang S, Ding SY. Lignin plays a negative role in the biochemical process for producing lignocellulosic biofuels. Curr Opin Biotechnol. 2014;27:38-45.

9. Swatloski RP, Spear SK, Holbrey JD, Rogers RD. Dissolution of cellulose with ionic liquids. J Am Chem Soc. 2002;124:4974-5.

10. Wahlström RM, Suurnäkki A. Enzymatic hydrolysis of lignocellulosic polysaccharides in the presence of ionic liquids. Green Chem. 2015;17:694-714.

11. Resch MG, Donohoe BS, Ciesielski PN, Nill JE, Magnusson L, Himmel ME, et al. Clean fractionation pretreatment reduces enzyme loadings for biomass saccharification and reveals the mechanism of free and cellulosomal enzyme synergy. ACS Sust Chem Eng. 2014;2:1377-87.

12. Chang RHY, Jang J, Wu KCW. Cellulase immobilized mesoporous silica nanocatalysts for efficient cellulose-to-glucose conversion. Green Chem. 2011;13:2844-50.

13. Lee YC, Chen CT, Chiu YT, Wu KCW. An effective cellulose-to-glucose-tofructose conversion sequence by using enzyme immobilized $\mathrm{Fe}_{3} \mathrm{O}_{4}$-loaded mesoporous silica nanoparticles as recyclable biocatalysts. ChemCatChem. 2013;5:2153-7.

14. Lee YC, Dutta S, Wu KCW. Integrated, cascading enzyme-/chemocatalytic cellulose conversion using catalysts based on mesoporous silica nanoparticles. ChemSusChem. 2014;7:3241-6.

15. Linares $N$, Serrano E, Rico M, Balu AM, Losada E, Luque R, et al. Incorporation of chemical functionalities in the framework of mesoporous silica. Chem Commun. 2011;47:9024-35.

16. Abraham RE, Verma ML, Barrow CJ, Puri M. Suitability of magnetic nanoparticles immobilized cellulases in enhancing enzymatic saccharification of pretreated hemp biomass. Biotech Biofuels. 2014;7:90-9.

17. You C, Chen H, Myung S, Sathitsuksanoh N, Ma H, Zhang XZ, et al. Enzymatic transformation of nonfood biomass to starch. Proc Natl Acad Sci USA. 2013;110:7182-7.

18. van den Brink J, de Vries RP. Fungal enzyme sets for plant polysaccharide degradation. Appl Microbiol Biotechnol. 2011;91:1477-92.

19. Beckham GT, Bomble YJ, Bayer EA, Himmel ME, Crowley MF. Applications of computational science for understanding enzymatic deconstruction of cellulose. Curr Opin Biotechnol. 2011;22:231-8.

20. Brunecky R, Alahuhta M, Xu Q, Donohoe BS, Crowley MF, Kataeva IA, et al. Revealing nature's cellulase diversity: The digestion mechanism of Caldicellulosiruptor bescii CelA. Science. 2013;342:1513-6.

Publish with ChemistryCentral and every
scientist can read your work free of charge
"Open access provides opportunities to our
colleagues in other parts of the globe, by allowing
anyone to view the content free of charge."
W. Jeffery Hurst, The Hershey Company.
- available free of charge to the entire scientific community
- peer reviewed and published immediately upon acceptance
- cited in PubMed and archived on PubMed Central
- yours - you keep the copyright
Submit your manuscript here:
htt://www.chemistrycentral.com/manuscript/

